\title{
Recomendaciones de actividad física y su relación con el rendimiento académico en adolescentes de la Región de Murcia \\ Physical activity recommendations and their relation with academic performance in adolescents from the Region of Murcia \\ Juan González Hernández, Alberto Portolés Ariño \\ Universidad de Murcia (España)
}

Resumen. Existe un gran interés por los beneficios psicológicos que reporta en las personas el realizar actividad física. Dicha actividad promueve mejoras en ciertos procesos cognitivos durante la adolescencia, sin embargo esta relación positiva no puede ser trasladada con la misma certeza a la relación entre el rendimiento académico y la actividad física. Identificar y acotar la existencia de relaciones entre el cumplimiento de las recomendaciones de práctica de actividad física y el rendimiento académico es el objetivo principal del presente estudio. Una muestra de 680 sujetos de la Región de Murcia con una edad media 14.55 años, cumplimentaron los ítems centrados en la actividad física del estudio internacional Health Behaviour in School-aged Children para abordar las variables objeto de estudio. Igualmente se registraron las calificaciones escolares de 4 trimestres consecutivos mediante un cuestionario de creación propia. Se encuentran resultados que acotan la existencia de relaciones entre la práctica de actividad física y el rendimiento académico. Realizar un mínimo de 30 minutos de actividad física diaria se relaciona directamente con mejores calificaciones escolares, en contraposición de la no práctica. Sin embargo, realizar un mínimo de 60 minutos diarios no señala la misma tendencia. El cumplimiento de las recomendaciones de práctica de actividad física y la duración de éstas se contemplar como un factor relacionado con el rendimiento académico de los adolescentes, siendo de este modo un aspecto importante a considerar en programas dirigidos a la intervención en el ámbito de la psicología y la salud.

Palabras clave. rendimiento académico, actividad física, salud, recomendaciones, adolescencia.

\begin{abstract}
There is a great interest in the psychological benefit generated by participation in physical activity. This activity fosters the improvement of some cognitive processes during adolescence, nevertheless there is not the same degree of certainty regarding the relation between physical activity and academic performance. The current study attempts to identify and delimit the association between compliance with physical activity recommendations and academic performance. A sample of 680 individuals from the region of Murcia, with an average age of 14.55 years , completed the items related to physical activity in the international study Health Behaviour in School-aged Children, in order to address the variables of interest. We recorded also student grades during four consecutive semesters using a questionnaire that we created for this purpose. We found an association between physical activity and academic performance. Engaging in 30 minutes of physical activity daily is closely related to better school grades, contrary to non-practice. However, the same tendency is not observed for 60 minutes of physical activity daily . Complying with physical activity recommendations, and to what extent, is considered a factor related to the teenagers' academic performance. As a result, it is an important aspect that we should consider for inclusion in health and psychology intervention programs.
\end{abstract}

Keywords: academic performance, physical activity, health, recommendations, adolescence.

\section{Introducción}

Es evidente que las funciones cognoscitivas son esenciales para enfrentarse con éxito a las demandas que trae consigo el aprendizaje. Desde su nacimiento, cada individuo aprende de los sucesos cotidianos que lo rodean, pero no es hasta que ingresa en la escuela donde el aprendizaje se vuelve formal y, la adquisición de la lectura y la escritura, así como los conocimientos básicos sobre matemáticas y ciencias, requieren de atender, comparar, diferenciar y buscar semejanzas para lograr asociar e integrar las nuevas ideas con las ya existentes (CastilloParra, Gómez \& Ostrosky-Solís, 2009).

Las habilidades cognitivas incluyen tanto las habilidades cognitivas básicas, tales como la función ejecutiva, la atención, la memoria, la comprensión verbal y el procesamiento de la información, así como las actitudes y creencias que influyen en el rendimiento académico, como la motivación, el autoconcepto, la satisfacción y el entorno escolar.

Sibley \& Etnier (2003) indican la existencia de una amplia evidencia científica que demuestra que la actividad física favorece y mejora los procesos cognitivos durante la niñez y la adolescencia. De hecho, concluyen señalando los beneficios que tiene para el desarrollo cognitivo el hecho de que los niños y adolescentes practiquen actividad física de manera regular. Del mismo modo, diferentes trabajos explican estos beneficios atendiendo a que la práctica de actividad física genera un aumento del flujo sanguíneo cerebral, cambios en la liberación de neurotransmisores, cambios estructurales en el sistema nervioso central y niveles elevados de excitación (Chaddock, et al., 2011; Halperin \& Healey, 2011).

Sin embargo la relación positiva que se da entre los procesos cognitivos y el rendimiento académico no se puede trasladar con la misma certeza científica a la relación entre rendimiento académico y

Fecha recepción: 14-01-15- Fecha envío revisores: 14-01-15- Fecha de aceptación: 16-10-15 Alberto Portolés Ariño albertoportol@hotmail.com actividad física, y a pesar de que la Organización Mundial de la Salud (1996) destacaba la importancia de la actividad física para la obtención de un mejor rendimiento académico, dicha relación sigue sin ser acotada de forma clara.

Por un lado, antes de analizar la relación entre las dos variables objeto de estudio, cumplimiento de las recomendaciones de actividad física y rendimiento académico, cabe contextualizar el momento actual en el que la práctica de actividad física se encuentra en la edad adolescente, ya que a pesar de que está socialmente arraigada la importancia de la actividad física, el sedentarismo es una problemática actual entre los adolescentes españoles, ocupando uno de los lugares más altos dentro del contexto europeo en cuanto a inactividad física se refiere (Moreno, et al. 2012; Noriega et al., 2015).

Las recomendaciones justificadas por Strong et al. (2005) son actualmente las más contempladas por los profesionales en el ámbito de la actividad física y deportiva señalando que los adolescentes deben participar en actividades físicas de al menos intensidad moderada durante 1 hora diaria (continuada o en periodos de 10 minutos a lo largo del día); por lo menos dos veces a la semana parte de estas actividades físicas deben ayudar a mejorar y mantener la fuerza muscular y la flexibilidad; y además los sujetos en edad escolar deben participar diariamente durante 60 minutos o más en actividades moderadas o vigorosas siendo placenteras, apropiadas e incluirán una gran variedad de actividades. Por otro lado el Ministerio de Educación y Ciencia junto con el de Sanidad y Consumo (2006) pautan una recomendación con una menor duración, de 30 minutos diarios.

De hecho, son muchos los estudios que abordan el cumplimiento de las recomendaciones mínimas para la salud de práctica de actividad física, y los cuales señalan dicha problemática (Janssen, et al. 2005; Neumark- Sztainer, Story, Hannan, Tharp \& Rex, 2003).

En el contexto español, Oviedo et al.,(2013) indican que los adolescentes dedican poco tiempo a la realización de AF, menor a 3 METs y en particular, las mujeres no alcanzan las mínimas recomendaciones sobre la práctica de AF en ninguno de los días evaluados. En un estudio 
anterior realizado en la ciudad de Huesca, si bien se evaluaron siete días, se determinó que sólo el 7.54\% de los chicos y el 3.44\% de las chicas cumplían las recomendaciones (Abarca-Sos, Zaragoza, Generelo \& Julián, 2010). Del mismo modo, Portolés (2014) señala como en la recomendación de realizar 60 minutos de actividad física diaria, el 77.77\% de la muestra total no cumplía dicha recomendación.

Por otro lado, en referencia al rendimiento académico González (2003) lleva a cabo una revisión donde identifica y clasifica los factores o condicionantes del rendimiento académico más investigados señalando tres factores generales: factores personales, la inteligencia y las aptitudes, la motivación, el autoconcepto y los hábitos, estrategias y estilos de aprendizaje, en lo que a los hábitos se refiere; factores escolares, el rendimiento anterior y el clima escolar; y factores psicosociales, nivel socioeconómico y cultural asociadas a la clase social, el medio educativo familiar, aspectos familiares, estilos de vida y variables socioambientales.

De este modo, el centro escolar es una parte esencial del entorno social de niños y adolescentes, en la medida que pasan mucho tiempo allí y que incluye una serie de influencias importantes que engloban las amistades, el profesorado y las oportunidades de realizar actividad física, encuadrada dentro de los factores psicosociales. La utilización del centro escolar como medio de promoción, la existencia de un entorno familiar y escolar favorable y concienciado, favorece que se propaguen estilos de vida activos y climas motivacionales elevados hacia la práctica de actividad física y deportiva (Martínez, Romero \& Delgado, 2010), favoreciendo de este modo a la salud psicológica del sujeto (TorneroQuiñones, Sierra-Robles, Carmona, \& Gago, 2015).

La relación diferencial entre ambas variables es abordada en varias revisiones bibliográficas internacionales. Howie \& Pate (2012) seleccionaron 125 artículos acerca de la influencia de la actividad física, tanto dentro como fuera del entorno escolar, sobre el rendimiento académico. Señalan que el 69\% de los estudios anteriores al 2007 muestran relaciones positivas entre la actividad física y el rendimiento académico, mientras que el porcentaje aumenta hasta $95 \%$ en los estudios posteriores al 2007. Además, el 78\% de las investigaciones en las que existe una intervención en la variable actividad física muestran relaciones positivas entre la actividad física y rendimiento académico antes del 2007 y del $83 \%$ en los años posteriores.

Trudeau \& Shephard(2008), en su revisión sugieren que un aumento de las actividades relacionadas con la actividad física no afecta negativamente al rendimiento académico, a pesar, de sustituir horas dedicadas anteriormente al estudio por actividades físicas, coincidiendo en este sentido con otros estudios (Bailey, 2006). En todos los estudios en los que se llevaba a cabo una intervención, encuentran señales que indican una relación positiva entre el rendimiento académico y la práctica de actividad física en el centro escolar, aunque algunas relaciones eranno significativas (Sallis, etal.1999), otras eran muy débiles (Ahamed, et al. 2007), y otras mostraban mejoras en algunas materias pero no en todas las analizadas según la intensidad de la práctica (Coe, Pivarnik, Womack, Reeves y Malina, 2006). En los estudios observacionales el $60 \%$ de los estudios analizados mostraba relaciones significativas positivas, el 30\% indicaba la no existencia de relaciones y el 10\% mostraba la existencia de relaciones inversas entre la actividad física escolar y el rendimiento académico.

Ramírez, Vinaccia \& Suarez (2004) también realizan una revisión destacando los numerosos beneficios de la actividad física para los procesos cognitivos y el rendimiento académico de los adolescentes. Concluyen que la actividad deportiva es un elemento central y fundamental en los programas de promoción de la salud para poblaciones infanto-juveniles con y sin patologías específicas como son las dificultades de aprendizaje.

El Centro para el Control y Prevención de Enfermedades de Estados Unidos (2010), llevó a cabo una profunda revisión bibliográfica con el objetivo de unificar todos los estudios que relacionan la actividad física y deportiva y el rendimiento académico. Tras una revisión inicial de 406 artículos con estudiantes entre 6 y 18 años, los 19 artículos que abordaban las actividades físicas organizadas por la escuela fuera del día escolar encontraron una o más asociaciones positivas.
En el contexto español, Ardoy et al. (2010), señalan tras una intervención basada fundamentalmente en el incremento del volumen (doble de sesiones de EF/semana) y la intensidad (doble de sesiones de EF/ semana de alta intensidad) que los adolescentes que completaron el programa mostraron mejores valores en capacidad cognitiva y rendimiento académico. En la misma línea, González \& Portolés (2014) muestran que el descenso de los niveles de práctica físico-deportiva, así como el aumento del consumo de sustancias nocivas repercuten de forma muy negativa en los adolescentes, además de influir en factores como el rendimiento y la motivación orientados al ámbito académico.

A pesar de que las revisiones anteriores señalaban (Howie \& Pate, 2012; Ramírez, et al., 2004; Trudeau \& Shephard, 2008) relaciones positivas entre actividad física y rendimiento académico, todas ellas coincidían en señalar la inconsistencia en dicha relación debido principalmente a la debilidad y escasa o nula significatividad de dichos resultados, las diferencias metodológicas para cuantificar tanto el rendimiento académico (calificaciones, tests estandarizados, observación...), como la actividad física (intensidad, duración, tipología...)

Por ello, se presenta como objetivo principal para el presente trabajo la necesidad de acotar las relaciones existentes entre la actividad física y el rendimiento académico en el contexto adolescente de la Región de Murcia, diferenciando según variables sociodemográficas. Del mismo modo, la revisión de la literatura existente permite extraer como hipótesis la existencia de relaciones positivas entre ambas variables de estudio.

\section{Metodología}

\section{Participantes}

La muestra está integrada por 680 estudiantes adolescentes matriculados en 6 centros públicos de la Región de Murcia, que fueron seleccionados a través de un muestreo aleatorio en función del área geográfica. La muestra tiene una edad media de 14.55 años (DT=1.21), distribuidos en 374 (55\%) sujetos del género masculino y 306 (45\%) del femenino y pertenecientes a los cuatro cursos de la ESO: $1^{\circ} \mathrm{ESO}$, 196 (28.8\%); $2^{\circ} \mathrm{ESO}, 101$ (14.9\%); $3^{\circ} \mathrm{ESO}, 224$ (32.9\%); $4^{\circ} \mathrm{ESO}, 159$ (23.4\%).

\section{Instrumentos}

Para cuantificar las dos variables objeto de estudio, actividad física y rendimiento académico se utilizan los siguientes cuestionarios:

-Recomendaciones de práctica de actividad física: selección de los ítems del cuestionario del estudio Health Behavior in School Aged Children (HBSC) (Moreno, Muñoz-Tinoco, Pérez y Sánchez-Queija, 2004) que concuerdan con los objetivos del presente trabajo, tal y como se realiza en estudios similares (Nuviala, et al. 2009; Ramos, Rivera, y Moreno, 2012). Los ítems seleccionados fueron: «en los últimos 7 días, ¿en cuántos días te sentiste físicamente activo/a durante un total de al menos 60 minutos por día? (Te preguntamos por el tiempo total; es decir, no es necesario que hayan sido 60 minutos seguidos, puedes sumar los distintos momentos del día en que realizabas algún tipo de actividad física); fuera del horario escolar: ¿Con quéfrecuencia realizas alguna actividad física en tu tiempo libre que haga que llegues a sudar o te falte el aliento?; fuera del horario escolar: ¿Cuántas horas a la semana sueles realizar alguna actividad física que haga que llegues a sudar o te falte el aliento durante tu tiempo libre?. Derivados de estos ítems se extraen las variables referentes al cumplimiento o no de las recomendaciones mínimas de actividad física, diferenciando entre las recomendaciones de 60' diarios de actividad física moderada (Strong, et al., 2005) y una menos restrictiva de cumplimiento de las recomendaciones de al menos 30' diarios (Estrategia NAOS, 2005).

-Rendimiento académico: se obtiene a partir de las calificaciones escolares en matemáticas, lengua castellana y literatura, inglés, educación física, sociales y ciencias de los alumnos en cuatro trimestres consecutivos, expresados mediante una nota media. Esta metodología de medición coincide con otros estudios que igualmente registran las calificaciones escolares medias (Córdoba, et al., 2012; Quevedo-Blasco \& Quevedo-Blasco, 2011) 


\section{Procedimiento}

Se establece contacto inicial con los centros resultantes del muestreo aleatorio para explicar los objetivos de la investigación, las pautas a seguir y para solicitar los permisos pertinentes. Tras el consentimiento de los responsables del centro, se procede al envío del correspondiente consentimiento informado a cada padre/madre/tutor. En dicho consentimiento se detalla el rol de sus hijos/as y el cumplimiento de privacidad y confidencialidad. La entrega final del cuestionario está sujeta a la presentación del consentimiento informado.

\section{Análisis Estadístico de los datos}

Este diseño se plantea con una metodología cuantitativa, siendo un diseño transversal descriptivo, por ello y atendiendo a los objetivos del presente estudio, se realizan análisis descriptivos básicos de tendencia central (media) y dispersión (desviación típica) para conocer detalladamente los datos de la muestra y las distribuciones de frecuencias; diferencias de medias, tablas de contingencia, pruebas Ty Anova. Para la realización de los análisis de datos se utiliza el programa estadístico SPSS, en su versión 19.0.

\section{Resultados}

Abordando inicialmente el análisis de resultados desde la variable actividad física, los porcentajes de cumplimiento de las recomendacio-

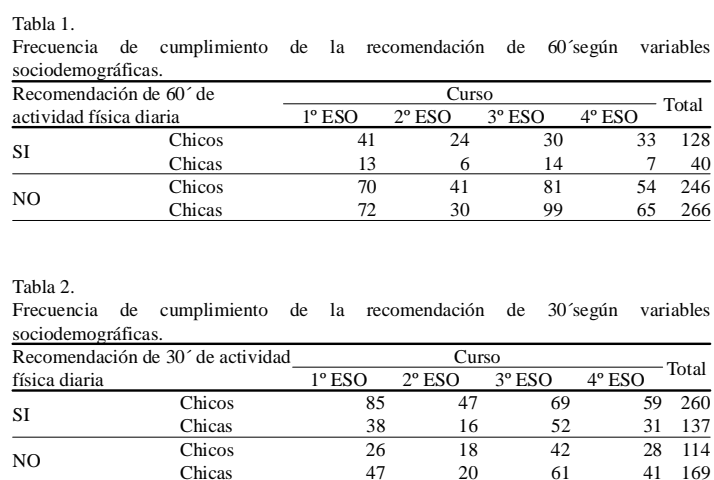

\begin{tabular}{|c|c|c|c|c|}
\hline 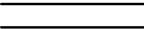 & Género & Media & D.T & $p$ \\
\hline \multirow{2}{*}{ Lengua } & Chicos & 5.32 & 1.84 & .000 \\
\hline & Chicas & 5.93 & 1.84 & \\
\hline \multirow{2}{*}{ Inglés } & Chicos & 5.44 & 2.02 & .001 \\
\hline & Chicas & 5.97 & 1.95 & \\
\hline \multirow{2}{*}{ Educación Física } & Chicos & 6.74 & 1.68 & .001 \\
\hline & Chicas & 6.29 & 1.88 & \\
\hline \multirow{2}{*}{ Sociales } & Chicos & 5.81 & 1.91 & .089 \\
\hline & Chicas & 6.07 & 2.06 & \\
\hline \multirow{2}{*}{ Matemáticas } & Chicos & 5.78 & 1.98 & .680 \\
\hline & Chicas & 5.85 & 1.94 & \\
\hline \multirow{2}{*}{ Ciencias } & Chicos & 5.99 & 1.97 & .146 \\
\hline & Chicas & 6.22 & 2.08 & \\
\hline \multicolumn{5}{|l|}{ Tabla 4.} \\
\hline \multicolumn{5}{|c|}{ Diferencia de medias del rendimiento académico según curso. } \\
\hline & & Media & D.T & $p$ \\
\hline \multirow{4}{*}{ Lengua } & $1^{\circ} \mathrm{ESO}$ & 5.60 & 2.01 & .001 \\
\hline & $2^{\circ} \mathrm{ESO}$ & 4.90 & 1.94 & \\
\hline & $3^{\circ} \mathrm{ESO}$ & 5.76 & 1.73 & \\
\hline & $4^{\circ} \mathrm{ESO}$ & 5.80 & 1.71 & \\
\hline \multirow{4}{*}{ Inglés } & $1^{\circ} \mathrm{ESO}$ & 5.55 & 2.20 & .000 \\
\hline & $2^{\circ} \mathrm{ESO}$ & 4.92 & 1.98 & \\
\hline & $3^{\circ} \mathrm{ESO}$ & 6.05 & 1.94 & \\
\hline & $4^{\circ} \mathrm{ESO}$ & 5.78 & 1.71 & \\
\hline \multirow{4}{*}{ Educación Física } & $1^{\circ} \mathrm{ESO}$ & 6.41 & 2.09 & .000 \\
\hline & $2^{\circ} \mathrm{ESO}$ & 5.79 & 1.99 & \\
\hline & $3^{\circ} \mathrm{ESO}$ & 6.65 & 1.41 & \\
\hline & $4^{\circ} \mathrm{ESO}$ & 7.00 & 1.54 & \\
\hline \multirow{4}{*}{ Sociales } & $1^{\circ} \mathrm{ESO}$ & 5.80 & 2.13 & .000 \\
\hline & $2^{\circ} \mathrm{ESO}$ & 5.06 & 1.95 & \\
\hline & $3^{\circ} \mathrm{ESO}$ & 6.34 & 1.79 & \\
\hline & $4^{\circ} \mathrm{ESO}$ & 6.06 & 1.88 & \\
\hline \multirow{4}{*}{ Matemáticas } & $1^{\circ} \mathrm{ESO}$ & 5.79 & 2.14 & .001 \\
\hline & $2^{\circ} \mathrm{ESO}$ & 5.10 & 2.14 & \\
\hline & $3^{\circ} \mathrm{ESO}$ & 6.02 & 1.86 & \\
\hline & $4^{\circ} \mathrm{ESO}$ & 6.00 & 1.63 & \\
\hline \multirow{4}{*}{ Ciencias } & $1^{\circ} \mathrm{ESO}$ & 5.92 & 2.30 & .000 \\
\hline & $2^{\circ} \mathrm{ESO}$ & 5.26 & 2.15 & \\
\hline & $3^{\circ} \mathrm{ESO}$ & 6.37 & 1.73 & \\
\hline & $4^{\circ} \mathrm{ESO}$ & 6.43 & 1.79 & \\
\hline
\end{tabular}

nes de práctica de actividad física en los adolescentes varía en función de la duración de dicha práctica. La actividad física realizada por los estudiantes y el cumplimiento de las recomendaciones mínimas muestra como el $24.7 \%$ de los sujetos de la muestra cumplen la recomendación de los 60 minutos de práctica diaria, por un 58.4\%, que cumplen la menos restrictiva de 30 minutos.

Según las variables sociodemográficas género y curso (Tabla 1 y Tabla 2), la recomendación de 60` es cumplida mayoritariamente por el género masculino, siendo en $1^{\circ}$ de la ESO donde la frecuencia es mayor $(n=41)$. Teniendo en cuenta la recomendación de los 30' aparece la misma tendencia, con el género masculino de $1^{\circ}$ de la ESO como principal cumplidor de dicha recomendación ( $\mathrm{n}=85$ ). Del mismo modo, las diferencias de medias en función del género señalan como los chicos cumplen significativamente en un mayor porcentaje la recomendación de 60 minutos $\left(\mathrm{t}_{678}=-6.551 ; p=.000\right)$ y de 30 minutos $\left(\mathrm{t}_{678}=-6.716 ; p=\right.$ .000) y en referencia al curso no aparecen diferencias significativas entre cursos para ninguna de las recomendaciones.

Por otro lado, el análisis de resultados centrado en la variable rendimiento académico señala en la tabla 3 las medias académicas de las materias registradas en función del género, pudiéndose observar que las chicas obtienen medias académicas más altas en todas las materias excepto en educación física, siendo significativa en este caso $\left(t_{678}=3.248\right.$; $p=.001)$, en lengua $\left(\mathrm{t}_{678}=-4.297 ; p=.000\right)$ y en inglés $\left(\mathrm{t}_{678}=-3.476 ; p=\right.$ $.001)$.

En la tabla 4, se observan las medias académicas según el curso, existiendo diferencias significativas en todas las materias: lengua $\left(\mathrm{F}_{676}=\right.$ 5.970; $\mathrm{p}=.000)$; inglés $\left(\mathrm{F}_{676}=8.048 ; \mathrm{p}=.000\right)$; educación física $\left(\mathrm{F}_{676}=\right.$ 10.561; $\mathrm{p}=.000)$; sociales $\left(\mathrm{F}_{676}=10.657 ; \mathrm{p}=.000\right)$; matemáticas $\left(\mathrm{F}_{676}=\right.$ 5.836; $\mathrm{p}=.000)$; $y$ ciencias $\left(\mathrm{F}_{676}=9.151 ; \mathrm{p}=.000\right)$.

La relación entre las dos variables objeto de estudio, rendimiento académico y el cumplimiento de las recomendaciones de práctica de actividad física, queda expuesto en las figuras 1 y 2. Los adolescentes que cumplen la recomendación más exigente, 60’, obtienen medias académicas inferiores a los que no la cumplen excepto en educación física, dónde los que sí realizan 60 minutos diarios de actividad física obtienen una puntuación media de 6.76 por un 6,48 de los que no. Sin embargo, una prueba $T$ para muestras independientes señala la no existencia de diferencias significativas para ninguna de las asignaturas. Considerando la recomendación menos restrictiva, 30', los resultados toman una dirección contraria a la anterior recomendación. Los adolescentes que cumplen la recomendación de realizar al menos 30 minutos diarios de actividad física obtienen resultados académicos superiores en todas las materias, siendo significativas en el caso de educación física $\left(t_{678}=6.847\right.$; $p=.000)$, ciencias $\left(\mathrm{t}_{678}=3.616 ; p=.000\right)$, matemáticas $\left(\mathrm{t}_{678}=2.223 ; p=\right.$ $.001)$ y sociales $\left(\mathrm{t}_{678}=3.227 ; p=.001\right)$.

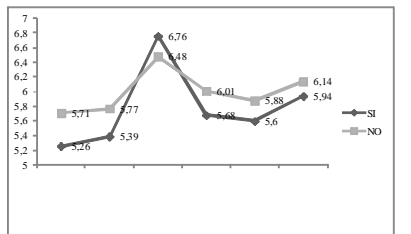

Figura 1. Relación diferencial entre el rendimiento académico y el cumplimiento de la recomendación de practicar 60’de actividad física.

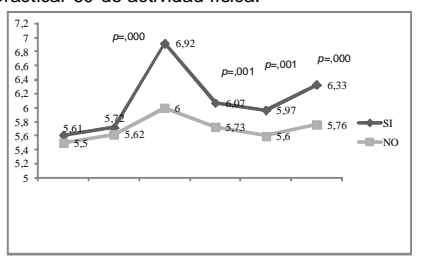

Figura 2. Relación diferencial entre el rendimiento académico y el cumplimiento de la recomendación de practicar $30^{\circ}$ de actividad física

\section{Discusión}

En primer lugar, con la variable de estudio actividad física, se opta al igual que en otros estudios (Montil, Barriopedro \& Oliván, 2005; Serra, 2009), por cuantificar el cumplimiento de las recomendaciones en 
función de dos criterios. El primero de ellos hace referencia a la práctica de actividad física diaria durante 60 minutos, y la segunda, menos restrictiva, aglutina a los practicantes diarios de 30 minutos de actividad física. Los resultados obtenidos señalan en la primera recomendación citada, un porcentaje de cumplimiento general para toda la muestra del $24.70 \%$, siendo muy similar al estudio de Janssen et al. (2005), que señala cómo un 33\% de la muestra cumple con las recomendaciones de realizar 60 minutos de actividad física. Laguna, Lara \& Aznar (2011), señalan un porcentaje superior, indicando que un $42.9 \%$ de su muestra cumple dicharecomendación. Considerando la segunda recomendación, el presente trabajo señala que un $58,40 \%$ de la muestra, realiza 30 minutos diarios de actividad física. Dicho porcentaje, está por encima del obtenido por Serra (2009), el cual señala un 41.52\%, y del obtenido por Montil et al. (2005) del 46.40\%.

En segundo lugar, tras el análisis de resultados de la variable rendimiento académico se observa como las adolescentes del género femenino obtienen puntuaciones mayores de rendimiento académico en todas las materias registradas, excepto en educación física, dónde los chicos obtienen puntuaciones más altas. Estos resultados concuerdan con estudios como el de Hernando, Oliva \& Pertegal (2012), que señalan igualmente un mayor rendimiento en el género femenino y un descenso de éste conforme aumenta la edad. Así pues, coincidimos plenamente con los resultados obtenidos por otros estudios (Córdoba, et al. 2011; Kovacs, et al. 2008; Niebla \& Hernández, 2007; Plazas, Aponte \& Ariza, 2006) observando que rendimiento académico en adolescentes y estudiantes de la ESO, es superior en el género femenino.

En referencia a las relaciones entre el cumplimiento de las recomendaciones de actividad física y el rendimiento académico, los resultados obtenidos señalan que en función de las características de la recomendación existe una dirección u otra en la relación diferencial establecida, por lo que la hipótesis planteada de la existencia de relaciones positivas entre ambas variables se cumple parcialmente, ya que dichas relaciones únicamente aparecen con la práctica diaria de 30 'de actividad física.

Contemplando la recomendación de al menos 60 minutos diarios de actividad física, los adolescentes que cumplen dicha recomendación obtienen menores medias académicas en todas las materias excepto en educación física, aunque no aparecen diferencias significativas. Sin embargo, contemplando la recomendación de 30 minutos diarios, los que si cumplen dicha recomendación, obtienen medias más altas en todas las materias, siendo significativas en el caso de matemáticas, ciencias, educaciones físicas y sociales. Los resultados del presente trabajo coinciden con lo que concluyen varias revisiones al respecto sobre la relación diferencial entre actividad física y rendimiento académico (Bailey, 2006; Capdevila, Bellmunt,\& Hernando, 2015; Howie \& Pate, 2012; Keeley \& Fox, 2009; Plaisance, Wilkinson, Miller \& Mahan,2015, Taras, 2005; Tomporowski, Davis, Miller \& Naglieri, 2008; Trudeau \& Shephard, 2008), señalando que a pesar de que se dan relaciones positivas, existe cierta inconsistencia, y que las diferentes metodologías aplicadas dificultan acotar dicha relación.

De este modo, se puede concluir para la presente muestra que realizar un mínimo de 30 minutos de actividad física diaria se relaciona directamente con mejores calificaciones escolares, en contraposición de los adolescentes que no practican. Sin embargo, aquellos adolescentes que realizan un mínimo de 60 minutos diarios no obtienen un mejor rendimiento académico. Por ello, futuros trabajos deberían profundizar en la duración de la práctica de AF ya que se contempla como un aspecto fundamental que determina el rendimiento académico de los adolescentes, siendo un factor importante para considerar en programas dirigidos a la intervención en este ámbito.

Los datos encontrados en este estudio deberían ser interpretados a la luz de algunas posibles limitaciones. En primer lugar, la adolescencia y la adultez temprana son etapas del desarrollo donde se producen cambios de tipo emocional y de formación de la identidad que pueden limitar en el tiempo la interpretación de los datos obtenidos. En segundo lugar, en estudios donde se realiza una administración colectiva de cuestionarios suele presentarse una alta tasa de respuestas azarosas, por lo que también podría haber sido interesante la aplicación de alguna escala de infrecuencia de respuesta o de deseabilidad social con la finalidad de detectar a los participantes con este tipo de perfil de respuestas. En tercer lugar, existe la limitación inherente a la aplicación de cualquier tipo de autoinforme, referida particularmente a la dificultad de algunos participantes para informar acerca de sus propios comportamientos, cogniciones y afectos.

\section{Referencias}

Abarca-Sos, A., Zaragoza, J., Generelo, E., \& Julián, J. A. (2010). Comportamientos sedentarios y patrones de actividad física en adolescentes. Revista Internacional de Medicina y Ciencias de la Actividad Física y el Deporte, 10(39), 410-427.

Ahamed, Y., Macdonald, H., Reed, K., Naylor, P., Liu-Ambrose, T., \& McKay, H. (2007). School-based physical activity does not compromise children's academic performance. Medicine and Science in Sports and Exercise, 39(2), 371-6.

Ardoy, D., Ortega, F., Fernández-Rodríguez, J., Chillón, P., Artero, E., España-Romero, V., Ortega, F. (2010). Educandompara mejorar el estado de forma física, estudio EDUFIT. Antecedentes, diseño, metodología y análisis del abandono/adhesión al estudio. Rev Esp Salud Pública, 84(2), 151-168.

Bailey, R. (2006). Physical Education and Sport in Schools: A Review of Benefits and Outcomes. Journal of School Health, 76(8), 397401.

Capdevila, A., Bellmunt, H., \& Hernando, C. (2015). Estilo de vida y rendimiento académico en adolescentes: comparación entre deportistas y no-deportistas. Retos: Nuevas tendencias en Educación Física, Deportes y Recreación, 27, 28-33

Castillo-Parra, G., Gómez, E., \& Ostrosky-Solís, F. (2009). Relaciones entre las Funciones Cognitivas y el nivel de Rendimiento Académico en niños. Revista Neuropsicología, Neuropsiquiatría y Neurociencias, 9(1), 41-54.

Centers for Disease Control and Prevention. (2010). The association between school based physical activity, including physical education, and academic performance. Atlanta: U.S. Department of Health and Human Services.

Chaddock, L., Pontifex, M. B., Hillman, C. H., \& Kramer, A. F. (2011). A review of the relation of aerobic fitness and physical activity to brain structure and function in children. Journal of the International Neuropsychological Society,17(06), 975-985.

Coe, D., Pivarnik, J., Womack C, J., Reeves, M., \& Malina, R. (2006). Effect of physical education and activity levels on academic achievement in children. Medicine and Science in Sports and Exercise, 38(8), 1515-9.

Córdoba, L., García, V., Luengo, L., Vizuete, M., \& Feu, S. (2012). How academic career and habits related to the school environmentinfluence on academic performance in the physical education subject. Retos. Nuevas tendencias en Educación Física, Deportey Recreación(21), 9-13.

Estrategia NAOS: Invertir la tendencia de la obesidad. Estrategia para la nutrición, actividad física y prevención de la obesidad. Madrid: Agencia española de SeguridadAlimentaria. Ministerio de Sanidad y Consumo, 2005.

González, C. (2003). Factores determinantes del bajo Rendimiento Académico en Educación Secundaria (Tesis Doctoral). Universidad Complutense de Madrid. Madrid.

González, J., \& Portolés, A. (2014). Actividad física extraescolar: relaciones con la motivación educativa, el rendimiento académico y conductas asociadas a la salud. Revista Iberoamericana de Psicología del Ejercicio y el Deporte, 9(1), 51-65.

Halperin, J. M., \& Healey, D. M. (2011). The influences of environmental enrichment, cognitive enhancement, and physical exercise on brain development: can we alter the developmental trajectory of ADHD?.Neuroscience \& Biobehavioral 
Reviews, 35(3), 621-634.

Hernando, A., Oliva, A., \& Pertegal, M. (2012). Variables familiares y rendimiento académico en la adolescencia. Estudios de Psicología, 33(1), 51-65.

Howie, E., \& Pate, R. (2012). Physical activity and academic achievement in children: A historical perspective. Journal of Sport and Health Science, 1, 160-169.

Janssen, I., Katzmarzyk, P., Boyce, W., Vereecken, C., Mulvihill, C., Roberts, C., Pickett, W. (2005). Comparison of overweight and obesity prevalence in school-aged youth from 34 countries and their relationships with physical activity and dietary patterns. Obesity Reviews, , 6, 123-132.

Keeley, T., \& Fox, K. (2009). The impact of physical activity and fitness on academic achievement and cognitive performance in children. International Review of Sport and Exercise Psychology, 2(2), 198-214.

Kovacs, F. M., Oliver-Frontera, M., Gil del Real, M. T., López Sanchez, J., Mufraggi, N., \& Palou, P. (2008). La influencia de los padres sobre el consumo de alcohol y tabaco y otros hábitos de los adolescentes de Palma de Mallorca en 2003. Revista Española de Salud, 82(6), 677-689.

Laguna, M., Lara, M. T., \& Aznar, S. (2011). Patrones de Actividad Física en función del género y los niveles de obesidad en población infantil española. Estudio EYHS. Revista de Psicología del Deporte, 20(2), 621-636.

Martínez,A., Romero, C., \& Delgado, M. (2010). Factores que inciden en la promoción de la actividad físico-deportiva en la escuela desde una perspectiva del profesorado. Cuadernos de Psicología del Deporte, 10(2), 57-75.

Ministerio de Sanidad y Consumo \& Ministerio de Educación y Ciencia.(2006). Actividad física y salud en la infancia y la adolescencia: Guía para todas las personas que participan en su educación. Madrid: Grafo, S.A.

Montil, M., Barriopedro, M. I., \& Oliván, J. (2005). El sedentarismo en la infancia: los niveles de actividad física en niños de la Comunidad Autónoma de Madrid. Apunts. Educació física i esports(82), 5-11.

Moreno, M.C., Muñoz-Tinoco, V., Pérez, P., Sánchez-Queija, I. (2004). Los adolescentes españoles y su salud. Madrid: Ministerio de Sanidad y Consumo.

Moreno, C., Ramos, P., Rivera, F., Jiménez-Iglesias, A., García Moya, I., Sanchez-Queija, I., . . . Granado, M. C. (2012). Las conductas relacionadas con la salud y el desarrollo de los adolescentes españoles: Resultados del estudio HBSC-2010 con chicos y chicas españoles de 11 a 18 años. Madrid: Ministerio de Sanidad, Servicios Sociaes e Igualdad.

Neumark-Sztainer, D., Story, M., Hannan, P. J., Tharp, T., \& Rex, J (2003). Factors associated with changes in physical activity: a cohort study of inactive adolescent girls. Archives of Pediatrics and Adolescent Medicine, 157(8), 803-810.

Niebla, J., \& Hernández, L. (2007). Variables que inciden en el rendimiento académico de adolescentes mexicanos. Revista latinoamericana de psicología, 39(3), 487-501.

Noriega, M. J., Jaén, P., Santamaría, A., Amigo, M. T., Antolín, O., Casuso, I., . . . De Rufino, P. M. (2015). Hábitos sedentarios en adolescentes escolarizados de Cantabria. Retos, 27, 3-7.

Nuviala Nuviala, A., Munguía Izquierdo, D., Fernández Martínez, A., Ruiz Juan, F., \& García Montes, M. E. (2009). Tipologías de ocupación del tiempo libre de adolescentes españoles: el caso de los participantes en actividades físicas organizadas. Journal of Human Sport \& Exercise, 4(1), 31-43.

Organización Mundial de la Salud.(1996). The Status of School Health. Génova: OMS.

Oviedo, G., Sánchez, J., Castro, R., Calvo, M., Sevilla, J. C., Iglesias, A., \& Guerra, M. (2013). Niveles de actividad física en población adolescente: estudio de caso. Retos: nuevas tendencias en educación física, deporte y recreación, 23, 43-47.
Plaisance, E. P., Wilkinson, L. L., Miller, K. E., \& Mahan, L. S. (2015). Factors Influencing the Accumulation of Recommended Physical Activity among Latinos in the Deep South of the United States. Retos: Nuevas tendencias en Educación Física, Deportes y Recreación, 27, 213-217.

Plazas, E. A., Aponte, R., \& Ariza, S. (2006). Relación entre estatus sociométrico, género y rendimiento académico. Psicología desde el Caribe, 17, 176-195.

Portolés, A. (2014). Orientación de metas y práctica de actividad física en adolescentes. Implicaciones sobre el rendimiento académicoyel burnout (Tesis Doctoral). Universidad de Murcia. Murcia.

Quevedo-Blasco, V. J., \& Quevedo-Blasco, R. (2011). Influencia del grado de somnolencia, cantidad y calidad de sueño sobre el rendimiento académico en adolescentes. International journal of clinical and health psychology, 11(1), 49-65.

Ramírez, W., Vinaccia, S., \& Suarez, G. R. (2004). El impacto de la actividad física y el deporte sobre la salud, la cognición, la socialización y el rendimiento académico: una revisión teórica. Revista de Estudios Sociales(18), 67-75.

Ramos, P., Rivera, F., \& Moreno, C. (2012). Benefits of the associative context in the leisure activities of Spanish adolescents. Infancia $y$ Aprendizaje, 35(3), 365-378.

Sallis, J., McKenzie, T., Kolody, B., Lewis, M., Marshall, S., \& Rosengard, P. (1999). Effects of health-related physical education on academic : project SPARK. Res Q Exerc Sport, 70(2), 127-34.

Serra, J. R. (2009). Factores que influencian la práctica de la actividad física en la población adolescente de la provincia de Huesca (Tesis Doctoral). Universidad de Zaragoza. Zaragoza.

Sibley, B., \& Etnier, J. (2003). The relationship between physical activity and cognition in children: A meta-analysis. Pediatric Exercise Science, 15, 243-256.

Strong, W. B., Malina, R. M., Blimkie, C. L., Daniels, S. R., Dishman, R. K., Gutin, B., Hergenroeder, M. D. (2005). Evidence based physical activity for school-age youth. Journal Pediatrics(146), 732-737.

Taras, H. (2005). Physical Activity and Student Performance at School. Journal of School Health, 75(6), 214-218.

Tomporowski, P., Davis, C., Miller, P., \& Naglieri, J. (2008). Exercise and Children's Intelligence, Cognition, andAcademic Achievement. Educational Psychology Review, 20,111-131.

Tornero-Quiñones, I., Sierra-Robles, Á., Carmona, J., \& Gago, J. (2015). Implicaciones didácticas para la mejora de la imagen corporal y las actitudes hacia la obesidad desde la Educación Física. Retos: Nuevas tendencias en Educación Física, Deportes y Recreación, 27, 146-151.

Trudeau, F., \& Shephard, R. (2008). Physical education, school physical activity, school sports and academic performance. International Journal of Behavioral Nutrition and PhysicalActivity, 5(10).

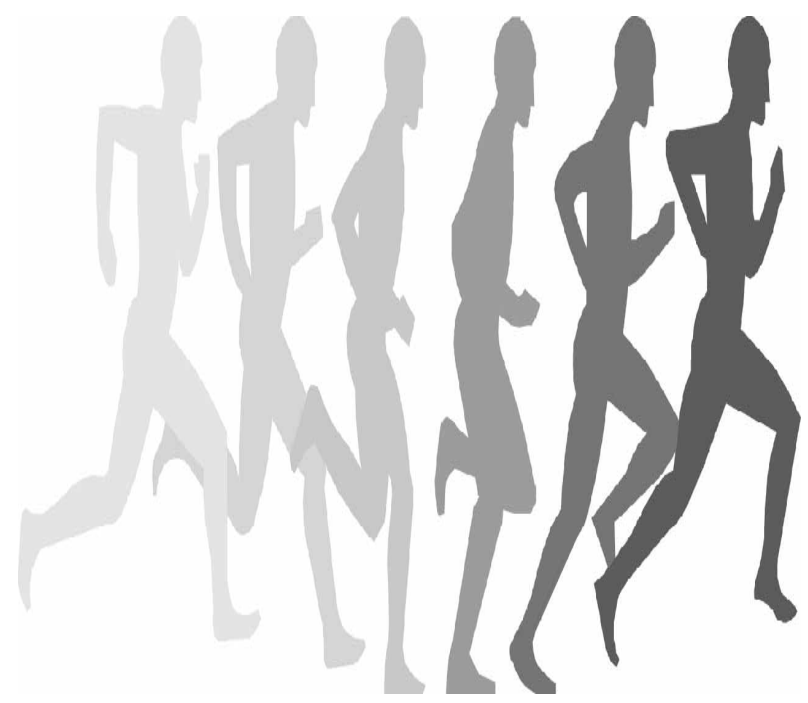

